In view of this experience, it is suggested that sugar chromatograms of biological material which it might be impractical to de-salt should be run in a neutral medium. We have found the $n$-propanol/ethyl acetate/water mixture described above highly suitable for separation of reducing sugars occurring in biological fluids.

\section{S. BAAR}

J. P. BULL

Medical Research Council

Industrial Injuries and Burns Research Unit, Birmingham Accident Hospital, Birmingham 15. May 9.

" Baar, S., and Bull, J. P., Lancet, 1, 978 (1952).

${ }^{2}$ Kennedy, E. P., and Barker, H. A., Anal. Chem., 23, 1033 (1951).

\section{Slow-Neutron Scattering Cross-Sections of Magnesium}

THE bound-atom and the coherent scattering cross-sections of magnesium have been given ${ }^{1}$ as $4 \cdot 2$ and $2 \cdot 4$ barns respectively, and it has been pointed out ${ }^{2,3}$ that these values are inconsistent with later measurements. In order to decrease the margin of uncertainty, we have re-determined the free-atom scattering cross-section.

The total cross-section of magnesium has been measured using the arrangement shown in Fig. 1, which selects neutrons in the energy-range $0.5-$ $1,000 \mathrm{eV}$. The number of neutrons scattered by the $2 \mathrm{gm} . / \mathrm{cm} .^{2}$ of $\mathrm{B}_{2} \mathrm{O}_{3}$ or $0.070 \mathrm{in}$. of polythene is the same. Thus, if we denote the counting-rates with and without the sample when the polythene (boron) is in position by $P_{8}\left(B_{\delta}\right)$ and $P(B)$ respectively, the ratio $\left(P_{8}-B_{8} /(P-B)\right.$ will be the transmission of the sample for those neutrons which are absorbed by the $2 \mathrm{gm}$. $/ \mathrm{cm}^{2}$ of $\mathrm{B}_{2} \mathrm{O}_{3}$. The calculated spectrum of these neutrons is shown in Fig. 2, and their calculated mean wave-length is $0 \cdot 145 \mathrm{~A} .(3 \cdot 9 \mathrm{eV}$.). Over the neutron energy-range covered by this spectrum, the absorption cross-section of magnesium is negligible $^{4}$ and the total cross-section is constant ${ }^{4}$, so that our measurement is of the free-atom scattering crosssection.

Measurements were carried out with samples of magnesium metal and its oxide. The oxygen total cross-section $^{4}$ was subtracted from the magnesium oxide cross-section. The results obtained were $3 \cdot 53 \pm$ 0.14 and $3.49 \pm 0.12$ barns from the magnesium and magnesium oxide measurements respectively. The mean value is $3.51 \pm 0.09$ barns. This yields a calculated bound-atom scattering cross-section of $3.82 \pm 0.10$ barns compared to $3 \cdot 8 \pm 0.2$ quoted in ref. 4.

The coherent scattering cross-section has been determined recently by the neutron diffraction ${ }^{2}$ and the mirror ${ }^{5}$ techniques, values of $3.4 \pm 0.14$ and $3 \cdot 7 \pm 0 \cdot 15$, respectively, being obtained. If the mean of these values is subtracted from our value for the bound-atom cross-section, a value of $0.27 \pm 0.14$
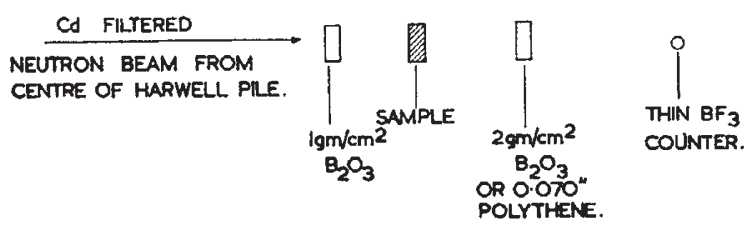

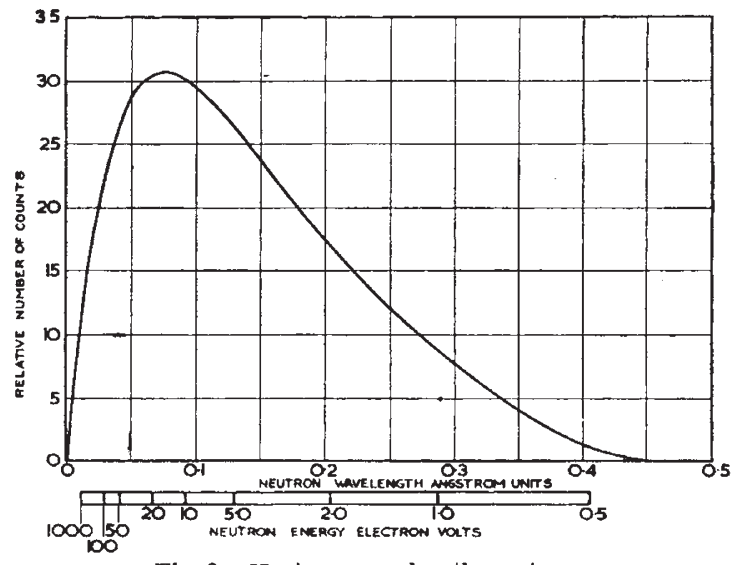

Fig. 2. Neutron wave-length spectrum

barn is obtained for the incoherent cross-section. This result is consistent with the direct measurements of Squires ${ }^{3}\left(0.11\right.$ barn) and Egelstaff ${ }^{6}(0.09 \pm 0.02$ barn) of the incoherent cross-section. Thus the values of the scattering cross-sections of magnesium are now in better agreement.

Atomic Energy Research Establishment,

P. A. Egelstaff

R. JACKSON

Harwell,

Nr. Didcot, Berks.

June 8.

1 Shull, C. G., and Wollan, E. O., Phys. Rev., 81, 527 (1951).

${ }^{2}$ Bacon, G. E., Acta Cryst., 5, 684 (1952).

a Squires, G. L., Proc. Roy. Soc., A, 212, 192 (1952).

4 U.S.A.E.C.U. 2040 (1952).

- Heindl, C., and Ruderman, I. W., Columabia Univ. (N.Y.) Dept. Physics, Quarterly Report $C U$ " 97 .

- Eigelstaff, P. A., A.E.R.E., N/R 1165 (1953).

\section{Photo-elastic Effect in Lead Nitrate}

IN an earlier communication ${ }^{1}$, it was reported that barium nitrate behaves in an exceptional manner in respect of its photo-elasticity. Since then, crystals of lead nitrate have also been studied, and differences between the stress-optical constants, as determined by \& Babinet compensator for the sodium D lines, are found to be:

$$
\left(q_{11}-q_{12}\right)=-19 \cdot 13 \times 10^{-13},\left(q_{11}-q_{12}\right)=-11.84 \times
$$$$
10^{-13} \text { and } q_{44}=-1.393 \times 10^{-13} \text { c.G.s. units. }
$$

These values are similar to those obtained in barium nitrate in being quite large. $\left(q_{11}-q_{12}\right)$ is different from $\left(q_{21}-q_{13}\right)$, as we should expect for the $T_{k}$ class of crystals to which lead nitrate belongs. The difference is 60 per cent of the lower value and is very large indeed. As in barium nitrate, in this substance also, the birefringence produced by pressure along a cube axis is nearly fourteen times that produced by an equal pressure along a cube diagonal. The photoelastic behaviour of lead nitrate is therefore to be regarded as highly anisotropic and very exceptional.

$$
\text { S. Bhagavantam }
$$

K. V. KRIShNa RaO

Physical Laboratories,

Osmania, University, Hyderabad. April 10.

${ }^{1}$ Nature, 162, 740 (1948). 